\title{
Effects of resveratrol on methotrexate-induced intestinal injury
}

\author{
Yulug $\mathrm{E}^{1}$, Turedi $\mathrm{S}^{1}$, Alver $\mathrm{A}^{2}$, Turedi $\mathrm{S}^{3}$, Kahraman $\mathrm{C}^{2}$ \\ Karadeniz Technical University, Faculty of Medicine, Department of Histology and Embryology, \\ Trabzon, Turkey. esinyulug@gmail.com
}

\begin{abstract}
BACKGROUND: Methotrexate (MTX) is an anticancer drug. Many studies have reported that MTX causes oxidative stress-associated damage in the small intestine. The purpose of this study was to investigate the possible protective effect of resveratrol (RES), an antioxidant, against MTX-induced damage in the small intestine. MATERIALS AND METHODS: Twenty-four Spraque Dawley rats were randomly divided into four groups; the control group, the RES group given $20 \mathrm{mg} / \mathrm{kg}$ RES for 10 days, the MTX group given single dose $30 \mathrm{mg} / \mathrm{kg}$ MTX, MTX+RES group given $20 \mathrm{mg} / \mathrm{kg}$ RES i.p. for 7 days and $30 \mathrm{mg} / \mathrm{kg}$ MTX i.p. on the 7 th day, RES being maintained for 3 further days. All rats were sacrificed on the 10th day, and small intestinal tissue was removed for histopathological and biochemical analysis. Additionally, mucosal apoptosis was analyzed using the TUNEL method. RESULTS: Histopathologically, villar fusion, atrophic villus epithelium, cystic expansion in crypts, hemorrhage and inflammatory cell infiltration were seen in the small intestine in the MTX group. In the MTX+RES group this histopathological damage decreased significantly. Apoptotic score was significantly higher in the MTX group and significantly lower in the MTX+RES group. Tissue malondialdehyde (MDA) level was significantly higher in the MTX group. Superoxide dismutase (SOD) activity was significantly decreased in the MTX group. The MDA level in the MTX+RES group decreased while SOD and catalase (CAT) activities rose, this was not statistically significant.

CONCLUSIONS: RES treatment may ameliorate MTX induced small intestine damage especially at histopathological level (Tab. 2, Fig. 2, Ref. 41). Text in PDF www.elis.sk.

KEY WORDS: methotrexate, resveratrol, rat, small intestine, oxidative stress.
\end{abstract}

\section{Introduction}

Methotrexate (MTX) is a cytotoxic chemotherapeutic agent widely used in leukemia and other malignancies. It is also used in non-oncological diseases, such as rheumatoid arthritis and psoriasis $(1,2)$. MTX is a folic acid antagonist and inhibits the enzyme dihydrofolate reductase (the essential enzyme in DNA and RNA synthesis) (3). The cytotoxic effects of MTX are not limited to cancer cells alone. It has side-effects on rapidly proliferating cells, such as crypts of gastrointestinal mucosa $(1,4)$. MTX damages the intestinal mucosa and causes intestinal flora to enter the circulation. It affects the entire gastrointestinal system and leads to conditions that may result in nausea, vomiting, diarrhea, ulceration and hemorrhage $(1,5)$. This reduces quality of life in chemotherapy treated patients.

The mechanism causing these side-effects of MTX has not yet been fully clarified. However, oxidative stress and neutrophil infil-

${ }^{1}$ Karadeniz Technical University, Faculty of Medicine, Department of Histology and Embryology, Trabzon, Turkey, ${ }^{2}$ Karadeniz Technical University, Faculty of Medicine, Department of Medical Biochemistry, Trabzon, Turkey, and ${ }^{3}$ Karadeniz Technical University, Faculty of Medicine, Department of Emergency Medicine, Trabzon, Turkey

Address for correspondence: E. Yulug, MD, PhD, Department of Histology and Embryology, Faculty of Medicine, Karadeniz Technical University, 61080, Trabzon, Turkey.

Phone: +90.462 .3777736 , Fax: +90.462 .3252270$ tration have recently been reported to play a role in the intestinal damage caused by MTX (6). MTX has been shown to significantly reduce antioxidant protein and enzyme levels and to increase the levels of oxidant markers such as malondialdehyde (MDA) (7, 8). Therefore researchers have studied various antioxidant agents in order to reduce the side-effects of MTX to a minimum $(9,10)$.

Resveratrol (trans-3,4',5-trihidroksi-stilben) (RES) is a polyphenol phytoalexin. It is found in various different plants, and particularly in grape, peanut and mulberry $(11,12)$. Research into the effects of RES has increased in recent years. Studies have emphasized its antioxidant, anti-inflammatory, antiplatelet, antiatherogenic and cancer inhibiting effects (13). RES scavenges reactive oxygen species (ROS), prevents damage to DNA and lipid peroxidation in the cell membrane (14).

The purpose of this study was to investigate the role of oxidative stress in MTX-associated small intestine injury and also to show the probable protective effects of RES, a known antioxidant, in small intestinal injury developing in association with MTX.

\section{Materials and methods}

\section{Animals}

Twenty-four healthy Spraque Dawley male rats (8 weeks old, weighing 200-250 g) were utilized in this study. Rats were kept in standard, controlled environments at a stable temperature (22 $\pm 2{ }^{\circ} \mathrm{C}$ ) in a 12:12-h light/dark cycle with access to standard rat 
chow and tap water ad libitum. All animals received human care according to the criteria outlined in the "Guide for the Care and Use of Laboratory Animals" published by the National Institutes of Health. The study was approved by the Institutional Animal Ethical Committee of the Karadeniz Technical University, Trabzon, Turkey.

\section{Study groups and Experimental design}

Rats were randomly divided into four groups and each group contained six rats. These were the control group, the RES group given $20 \mathrm{mg} / \mathrm{kg}$ of resveratrol (Resveratrol, R5010-500 mg, Sigma-Aldrich,St.Louis, MO, USA) intraperitoneally (i.p.) over 10 days, the methotrexate (Methotrexate, Kocak Farma, Tekirdag, Turkey) group given $30 \mathrm{mg} / \mathrm{kg}$ i.p. on the 7 th day of the experiment, and the MTX+RES group given $20 \mathrm{mg} / \mathrm{kg}$ RES i.p. for 7 days and $30 \mathrm{mg} / \mathrm{kg}$ MTX i.p. on the 7th day and then $20 \mathrm{mg} / \mathrm{kg}$ RES for another 3 days. At the end of the 10th day laparotomy was performed in all rats under ketamine hydrochloride (Ketalar, Pfizer, Istanbul, Turkey) anesthesia. The abdominal cavity was opened, and a $5-\mathrm{cm}$ intestinal segment from a point $5 \mathrm{~cm}$ proximal to the ileocecal ligament was rapidly dissected. Half the tissue was fixed in $10 \%$ formalin for histopathological evaluation, while the other half was placed in an eppendorf tube and stored at $-80{ }^{\circ} \mathrm{C}$ for biochemical investigation. Immediately afterwards, $3 \mathrm{cc}$ blood specimens were placed in an EDTA containing tube, centrifuged and stored at $-80{ }^{\circ} \mathrm{C}$ for biochemical investigation.

\section{Biochemical analysis}

Determination of MDA

A piece of ileum tissue was used to measure malondialdehyde (MDA) levels. The sample was minced and homogenized in an ice-cold $1.15 \% \mathrm{KCl}$ solution containing $0.50 \mathrm{~mL} / \mathrm{L}$ Triton X-100 using an Ultra-Turrax T25 homogenizer. MDA levels in testis samples were determined as MDA concentration by the method of Mihara and Uchiyama (15). Briefly, $0.5 \mathrm{~mL}$ of homogenate was mixed with $3 \mathrm{~mL}$ of $1 \% \mathrm{H}_{3} \mathrm{PO} 4$. After adding $1 \mathrm{~mL}$ of $0.67 \%$ thiobarbituric acid, the mixture was heated in boiling water for 45 min. The formed color was extracted into n-butanol. The mixture was centrifuged at $4000 \mathrm{rpm}$ for $10 \mathrm{~min}$ at room temperature. Absorbance of the organic layer was read at $532 \mathrm{~nm}$. Tetramethoxypropane was used as a standard, and MDA levels were calculated as nanomoles per gram of wet tissue.

\section{Determination of SOD and CAT Activity}

Superoxide dismutase (SOD) and catalase (CAT) activities were determined in the remaining part of the ileum tissue. The sample was minced and homogenized in an ice-cold Tris-HCL buffer ( $50 \mathrm{mM}$, pH 7.4) containing $0.50 \mathrm{~mL} / \mathrm{L}$ Triton X-100. SOD activities were measured by degree of the inhibition rate of nitroblue tetrazolium reduction in the xanthine-xanthineoxidase system (16). Enzyme activity leading to $50 \%$ inhibition was accepted as one unit and results were expressed as U/g tissue protein. CAT activity was determined by the method of Aebi (17) and results are expressed as $\mathrm{k} / \mathrm{g}$ protein ( $\mathrm{k}$, rate constant). Protein concentrations were determined according to Bradford's method (18).

\section{Histopathological evaluation}

Ileum tissues were dehydrated, and embedded in paraffin for light microscopic evaluation. Sections $5 \mu \mathrm{m}$ in thickness were taken and stained with hematoxylin-eosin (H\&E). The sections were analyzed under a light microscope (Olympus BX-51; Olympus, Tokyo, Japan). Five different fields were evaluated at a magnification of X200 in ileum slides from all groups. Each section was scored semi-quantitatively from 0 to 3 (0: none; 1 : mild; 2 : moderate and 3: severe) according to defined criteria: Degeneration of the apical surface epithelium, villar fusion, hemorrhage and inflammatory cell infiltration. The mean histopathologic score was calculated for each group (19).

\section{TUNEL assay}

We evaluated the levels of apoptosis in ileum using a standard terminal deoxynucleotidyl transferase (TdT) deoxyuridine triphosphate nick end labeling assay (TUNEL) method. TUNEL staining of sections was performed using an in situ cell death detection kit, AP (Roche, Mannheim, Germany), in accordance with the manufacturer's instructions. The sections were incubated in a humidified chamber with the TUNEL reaction mixture. The color was then developed with 3,3'-diaminobenzidine (DAB) including kit (DAB, Sigma, St. Louis, MO, USA). The TUNEL staining cells appear as brown and other cells nuclei appear as blue stains (20). TUNEL-stained histologic slides were examined by a blinded histologist, a score on the scale of $0-4$ was determined. Under that classification, score 0 represented only a few apoptotic TUNEL positive nuclei observed in the villous epithelium, score 1 observed apoptotic nucleus clusters at the villous tips, score 2 observed apoptotic nuclei in all villi but not in crypts, score 3 observed apoptotic nuclei in villi and crypts, score 4 represented apoptotic nuclei in all layers (21).

\section{Statistical analysis}

All statistical analyses were performed using the SPSS version 13.0 Med Calc 12.3 statistical software. All data were presented as mean $( \pm)$ standard deviation (SD). Kruskal Wallis analysis of variance was used to compare differences between group parameters. Dual comparisons between groups exhibiting significant values were evaluated with a Mann-Whitney U-test- with corrected Bonferroni test. Statistical significance was accepted for all tests at $\mathrm{p}<0.05$.

\section{Results}

\section{Clinical signs}

Rats in all groups survived during the experiment. Diarrhea was observed in all rats in the MTX group following MTX administration. While diarrhea was not observed in the control and RES groups, it was seen in two rats in the MTX+RES group. However, this was not as severe as that in the MTX group. Body weight $(194.90 \mathrm{~g})$ decreased in the MTX group compared to control and RES group (227.26g and $221.40 \mathrm{~g}$, respectively), body weight was greater in the MTX+RES group (198.8 g) compared to the MTX group, but the differences were not statistically significant $(\mathrm{p}>0.05)$. 


\section{$676-680$}

\section{Biochemical analysis results}

Ileal MDA level, SOD and CAT activities are shown in Table 1. Ileal MDA levels were significantly higher in the MTX group compared to the control group ( $p<0.05$ ). MDA levels in the MTX+RES group were decreased compared to the MTX group, but there was no statistical significance. Ileal SOD activity was significantly lower in the MTX group compared to the control group $(\mathrm{p}<0.05)$. Ileal CAT activity was lower in the MTX group compared to the control group, but there was no statistical significance. Although SOD and CAT activities increased in the MTX + RES group compared to the MTX group, that increase was not statistically significant $(\mathrm{p}>0.05)$.

\section{Histopathological findings}

Ileal damage score values on the basis of parameters analysis are shown in Table 2. The small intestine was evaluated macroscopically. No color change was observed in the control, RES and MTX+RES groups. In the MTX group, however, a dark, blue/purple color was seen. At evaluation under light microscopy, control and RES groups ileal tissue exhibited normal histological findings (Figs 1a, b). Examination of the MTX group revealed vacuolization and shedding in ileal epithelial cells and shortening, blunting and fusion in villi. Hemorrhage was present in all layers, particularly in the lamina propria. A decrease in crypts, epithelial loss and a cystic appearance were observed. Loss of goblet cells and widespread inflammatory cell infiltration in mucosa were observed (Fig. 1c). Ileal damage score values on the basis of parameters analysis were significantly increased in MTX group compared to the control group $(\mathrm{p}<0.05)$. In MTX+RES group, villous architecture was close to normal, and villus epithelial cells exhibited a regular arrangement. Goblet cells were present among the epithelial cells (Fig. 1d). Degeneration of the apical surface epithelium and hemorrhage were significantly decreased compared to the MTX group $(\mathrm{p}<0.05)$. Moderate inflammatory cell infiltration was observed in the mucosa.

Tab. 1. Biochemical analysis of ileum tissues for each group.

\begin{tabular}{lccc}
\hline Groups & \multicolumn{3}{c}{ Parameters } \\
\cline { 2 - 4 } & MDA(nmol/g) & SOD $(\mathrm{U} / \mathrm{g}$ tissue $)$ & CAT $(\mathrm{k} / \mathrm{g})$ \\
\hline Control & $114.65 \pm 23.20$ & $279.65 \pm 29.99$ & $0.65 \pm 0.09$ \\
RES & $110.65 \pm 26.90$ & $284.09 \pm 77.77$ & $0.61 \pm 0.08$ \\
MTX & $182.31 \pm 21.82^{*}$ & $199.24 \pm 19.19^{*}$ & $0.47 \pm 0.05$ \\
MTX+RES & $139.79 \pm 23.41$ & $226.17 \pm 44.26$ & $0.53 \pm 0.12$ \\
\hline MDA-malondialdehyde, SOD - superoxide dismutase, CAT - catalase, RES - Res- \\
veratrol, MTX - Methotrexate, * $\mathrm{p}<0.05$ vs Control
\end{tabular}

\section{Evaluation of TUNEL staining}

Apoptotic score values of the study groups' ileal tissues are shown in Table 2. A few apoptotic cells were observed in the villus tips in the control and RES groups (Figs 2 a, b). Examination of the MTX group revealed wide spread apoptotic cells in all ileal layers (Fig. 2c). Apoptotic score was significantly higher in the MTX group compared to the control group $(\mathrm{p}<0.05)$. In the MTX+RES group, however, apoptotic cells were restricted to the villi (Fig. 2d). In the MTX+ RES group, apoptotic score was significantly lower compared to the MTX group $(\mathrm{p}<0.05)$.

\section{Discussion}

MTX is one of the most frequently used anti-metabolitic agents in clinical oncology. Clinical use is frequently accompanied by diarrhea and gastrointestinal symptoms $(22,23)$. Ileal injury developing with the administration of MTX has been identified in the literature $(10,23,24,25)$. Previous studies have shown that a single dose of MTX is sufficient in the development of ileal damage (24), together with villous atrophy, flattening of the epithelium, crypt loss and restructuring of the crypts $(9,26)$. Damage evaluation in intestinal mucosal revealed damage the $72 \mathrm{nd} h$ following MTX administration (22). In this study we evaluated ileal damage histopathologically and biochemically at the end of the 3rd day after MTX administration.

MTX-induced organ toxicity mechanism has not been completely clarified. Recent studies show that the side-effects of antitumor drugs may be associated with free oxygen radicals and hydrogen peroxide $(9,27)$. Damage induced by the administration of MTX is characterized by inflammatory cell response $(9,22)$. Inflammatory cells activated by pro-cytokines lead to excessive free oxygen radical production, and that increases MDA, a product of lipid peroxidation (28). In our study, lipid peroxidation level in the MTX group exhibited a pronounced increase compared with the control and RES groups. The rise in MDA level may be attributed to tissue damage and oxygen defense mechanisms being insufficient in the prevention of free radical formation $(6,9)$. In the MTX +RES group, we observed a decrease in lipid peroxidation levels compared to the MTX group. This may be due to oxidation mechanisms becoming activated and binding free radicals.

Various enzymes play a role in oxidative stress and are effective in the protective mechanism against oxidative damage. These include enzymes such as SOD, CAT and glutathione peroxidase. These convert free oxygen radicals and reactive oxygen surfaces into non-radical products (29). SOD is a powerful antioxidant en-

Tab. 2. The histological damage score on the basis of analysis parameters and apoptotic score in control, RES, MTX, MTX+RES groups.

\begin{tabular}{|c|c|c|c|c|c|}
\hline \multirow[b]{2}{*}{ Groups } & \multicolumn{5}{|c|}{ Parameters } \\
\hline & Inflammatory cell infiltration & Villar fusion & $\begin{array}{l}\text { Degeneration of the apical } \\
\text { surface epithelium }\end{array}$ & Hemorrhage & Apoptotic score \\
\hline Control & $0.33 \pm 0.51$ & $0.16 \pm 0.4$ & $0.16 \pm 0.4$ & $0.16 \pm 0.4$ & $0.16 \pm 0.4$ \\
\hline RES & $0.50 \pm 0.54$ & $0.50 \pm 0.54$ & $0.16 \pm 0.4$ & $0.33 \pm 0.51$ & $0.3 \pm 0.51$ \\
\hline MTX & $2.5 \pm 0.54 *$ & $2.5 \pm 0.54 *$ & $2.66 \pm 0.51 *$ & $2.33 \pm 0.51 *$ & $3.66 \pm 0.51 *$ \\
\hline MTX+RES & $1.66 \pm 0.51 *$ & $1.33 \pm 0.51 *$ & $1.33 \pm 0.51 * * *$ & $1.16 \pm 0.4 * * *$ & $1.83 \pm 0.75 * * *$ \\
\hline
\end{tabular}

RES - resveratrol, MTX - methotrexate, $* \mathrm{p}<0.05$ vs Control, $* * \mathrm{p}<0.05$ vs MTX group 


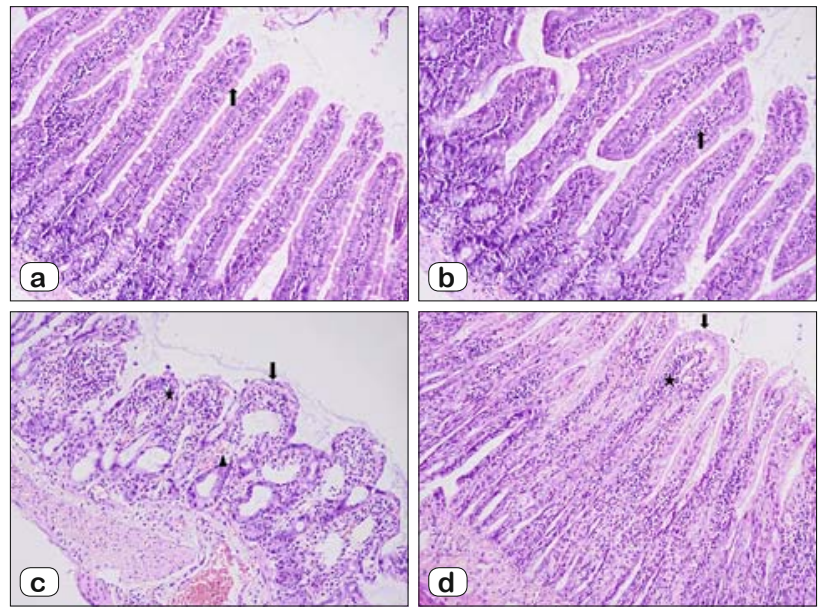

Fig. 1. Light microscopic sections of ileum tissues in different groups. a, b Normal villous architecture (thick arrow) was observed in control group and RES groups. $c$ In MTX group, villus epithelial degeneration (thick arrow), a cystic appearance in the crypts, severe inflammatory cell infiltration (star) and hemorrhage (arrow head) were observed. d In MTX+RES group, regeneration in epithelial cells in villi (thick arrow), crypts and presence of goblet cells, moderate inflammatory cell infiltration (star) and slight hemorrhage were observed (H\&E x200).
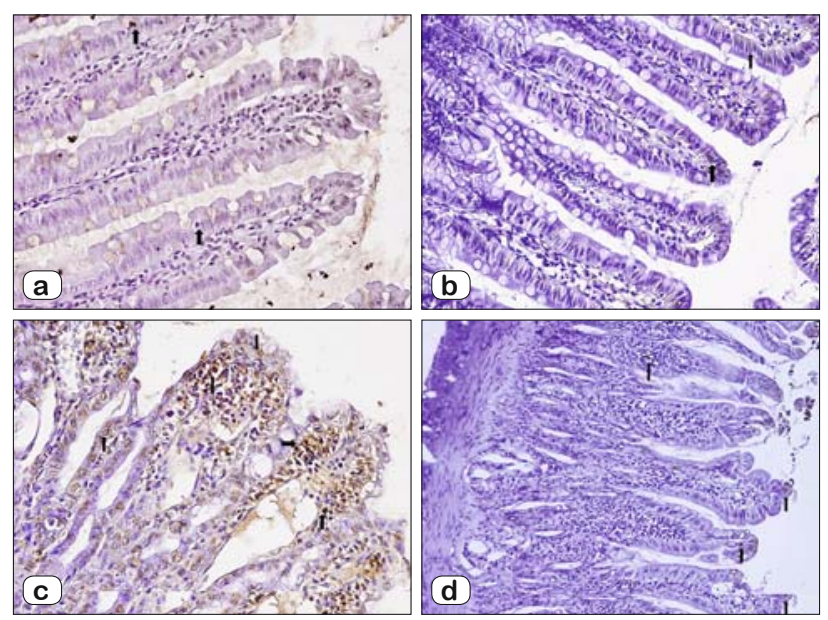

Fig. 2. TUNEL staining of ileum tissues in different groups. a, b In Control and RES group, respectively, a few TUNEL positive apoptotic cells were observed in villus tips (thick arrow), c In MTX group, apoptotic cells (thick arrow) were present in all layers of ileal tissues, $d$ In MTX+RES group, apoptotic cells (thick arrow) were only present in villous architecture (TUNEL $\mathrm{x200}$ ).

zyme that protects cells against reactive oxygen radicals. It does this by catalyzing the conversion of superoxide radicals to hydrogen peroxide (30). CAT is an antioxidant enzyme that consumes peroxisome hydrogen peroxide and protects the cells against oxidative damage (31). Cetin et al (32) observed an increase in MDA levels in hepatic oxidative damage induced by MTX, and also a decrease in SOD and CAT levels. Vardi et al (10) reported similar findings in MTX administration-associated small intestine damage. In our study, SOD and CAT levels were lower in the MTX group compared to the control group. The decrease in SOD and
CAT levels may be due to a decreased or increased consumption in the synthesis of antioxidant enzymes. There was a rise in SOD and CAT levels in the MTX+RES. With these results, we think that RES can regulate enzymatic activity in small intestinal damage.

In our MTX group we determined atrophy in the villi and crypts. We also observed that crypts have been replaced by cystlike structures and there was a decrease in goblet cells. Histopathological findings in the MTX group were compatible with findings from previous studies $(10,33,34)$. In the MTX+ RES group there was a pronounced improvement in villus structure compared to the MTX group; the appearance of the villous epithelium and goblet cells was close to normal, crypts were clearly visible, the cystic structure had disappeared and there was a pronounced decrease in hemorrhage. In terms of the pathology of villous degeneration, Gao et al (35) reported that MTX inhibits de novo purine and thymidine kinase synthesis, and that this effect also suppresses cell proliferation in the intestinal epithelial cells and crypts. MTX inhibits the dihydrofolate reductase enzyme. Dihydrofolate reductase is an enzyme essential for DNA synthesis. In this way, DNA synthesis is impaired (3) and apoptosis takes place in the cells $(3,36)$. While elevated Bax protein has been determined in MTX administrations, the level of Bcl 2 protein decreases (26). Bax protein stimulates leakage of cytochrome c from the mitochondria into cytoplasm, and this process activates apoptotic proteases. Additionally, production of reactive oxygen radicals such as superoxide radical gives rise to apoptotic cell death (9). Also, due to its antioxidant property, like vitamins C and E, RES also prevents cell death induced by oxidized lipoproteins (37). In our study, while widespread apoptosis was observed in all ileal layers in the MTX group, apoptotic cells were restricted to the villi in the RES treatment group. RES therapy was accompanied by a significant improvement in ileal morphology and a significant decrease in apoptosis compared to the MTX group. Since RES is both a free radical scavenger and also activates antioxidant enzymes, it has been described as a potent antioxidant $(38,39)$. RES inhibits the peroxidation of membrane lipids (37). Previous studies have shown positive effects of RES therapy in a small intestine model with induced ischemia-reperfusion damage $(40,41)$.

In conclusion, our study showed that oxidative stress plays an important role in the pathogenesis of MTX-induced intestinal damage. The powerful antioxidant RES given before MTX treatment has a protective effect on MTX-induced intestinal damage and apoptotic cell death in rats. This beneficial effect of RES might be due to its anti-oxidant properties.

\section{References}

1. Kolli VK, Abraham P, Rabi S. Methotrexate-induced nitrosative stress may play a critical role in small intestinal damage in the rat. Arch Toxicol 2008; 82: 763-770.

2. Pincus T, Yazici Y, Sokka T, Aletaha D, Smolen JS. Methotrexate as the "anchor drug" for the treatment of early rheumatoid arthritis. Clin Exp Rheumatol 2003; 21: 179-185.

3. Heenen M, Laporte M, de Graef C. Methotrexate induces apoptotic cell death in human keratinocytes. Arch Dermatol Res 1998; 290: 240-245. 


\section{$676-680$}

4. Naruhashi K, Nadai M, Nakao M, Suzuki N, Nabeshima T, Hasegawa T. Changes in absorptive function of rat intestine injured by methotrexate. Clin Exp Pharmacol Physiol 2000; 27: 980-986.

5. Jolivet J, Cowan KH, Curt GA, Clendeninn NJ, Chabner BA. The pharmacology and clinical use of methotrexate. N Engl J Med 1983; 309: 1094-1104.

6. Miyazono Y, Gao F, Horie T. Oxidative stress contributes to methotrexate-induced small intestinal toxicity in rats. Scand J Gastroenterol 2004; 39: 1119-1127.

7. Gulgun M, Erdem O, Oztas E et al. Proanthocyanidin prevents methot rexate-induced intest inal damage and oxidative stress. Exp Toxicol Pathol 2010; 62: 109-115.

8. Kesik V, Uysal B, Kurt B, Kismet E, Koseoglu V. Ozone ameliorates methotrexate-induced intestinal injury in rats. Cancer Biol Ther 2009; 8: $1623-1628$.

9. Jahovic N, Cevik H, Sehirli AO. Melatonin prevents methotrexate induced hepatorenal oxidative injury in rats. J Pineal Res 2003; 34: 282-287.

10. Vardi N, Parlakpinar H, Ozturk F et al. Potent protective effect of apricot and beta-carotene on methotrexate- induced intestinal oxidative damage in rats. Food Chem Toxicol 2008; 46: 3015-3022.

11. Krasnow MN, Murphy TM. Polyphenol glucosylating activity in cell suspension of grapes (Vitis vinifera). JAgric Food Chem 2004; 52: 3467-3472.

12. Caruso F, Tanski J, Villegas-Estrada A, Rossi M. Structural basis for antioxidant activity of trans-resveratrol: ab initio calculations and crystal and molecular structure. J Agric Food Chem 2004; 52: 7279-7285.

13. Ignatowicz E, Baer-Dubowska W. Resveratrol, a natural chemopreventive agent against degenerative diseases. Pol J Pharmacol 2001; 53: 557-569.

14. de la Lastra CA, Villegas I. Resveratrol as an antioxidant and prooxidant agent: mechanisms and clinical implications. Biochem Soc Trans 2007; 35: 1156-1160.

15. Mihara M, Uchiyama M. Determination of malondialdehyde precursor in tissues by thiobarbituricacid test. Anal Biochem 1978; 86: 271-278.

16. Sun Y, Oberley LW, Li Y. A simple method for clinical assay of superoxide dismutase. Clin Chem 1988; 34: 497-500.

17. Aebi H. Catalase. In: Bergmeyer HU (Ed). Methods of enzymaticanalysis. New York: Academic Press, 1974: 673-677.

18. Bradford MM. A rapid and sensitive method for the quantitation of microgram quantities of protein utilizing the principle of protein-dye binding. Anal Biochem 1976; 72: 248-254.

19. Gunduz A, Turkmen S, Turedi $S$ et al.Time-dependent variations in ischemia-modified albumin levels in mesenteric ischemia. Acad Emerg Med 2009; 16: 539-543.

20. Yuluğ E, Tekinbas $\mathbf{C}$, Ulusoy $\mathbf{H}$ et al.The effects of oxidative stress on the liver and ileum in rats caused by one-lung ventilation. J Surg Res 2006; 139: 253-260.

21. Jilling T, Lu J, Jackson M, Caplan MS. Intestinal epithelial apoptosis initiates gross bowel necrosis in an experimental rat model of neonatal necrotizing enterocolitis Pediatr Res 2004; 55: 622-629.

22. Soares PM, Lopes LO, Mota JM, Belarmino-Filho JN, Ribeiro RA, Souza MH. Methotrexate-induced intestinal mucositis delays gastric emptying and gastrointestinal transit of liquids in awake rats. Arq Gastroenterol 2011; 48: 80-85.

23. Sener G, Ekşioğlu-Demiralp E, Cetiner M, Ercan F, Sirvanci S, Gedik N. L-carnitine ameliorates methotrexate-induced oxidative organ injury and inhibits leukocyte death. Cell Biol Toxicol 2006; 22: 47-60.
24. Taminiau J, Gall DG, Hamilton JR. Response of the rat small-intestine epithelium to methotrexate. Gut 1980; 21: 486-492.

25. Cetiner M, Sener G, Sehirli AO et al. Taurine protects against methotrexate-induced toxicity and inhibits leukocyte death. Toxicol Appl Pharmacol 2005; 209: 39-50.

26. Koppelmann T, Pollak Y, Mogilner J, Bejar J, Coran AG, Sukhotnik I. Dietary L-arginine supplementation reduces Methotrexate-induced intestinal mucosal injury in rat. BMC Gastroenterol 2012; 12: 41.

27. McGinnes JE, Proctor PH, Demopoulos HB, Hokanson JA, Kirkpatrick DS. Amelioration of cis-platinum nephrotoxicity by orgotein (superoxide dismutase). Physiol Chem Phys 1978; 10: 267-277.

28. Guneli E, Cavdar Z, Islekel H et al. Erythropoietin protects the intestine against ischemia/reperfusion injury in rats. Mol Med 2007; 13: 509-517.

29. Fujii, J, Iuchi Y, Matsuki S, Ishii T. Cooperative function of antioxidant and redox systems against oxidative stress in male reproductive tissues. Asian J Androl 2003; 5: 231-242.

30. Celino F T, Yamaguchi S, Miura C et al. Tolerance of Spermatogonia to Oxidative Stress Is Due to High Levels of $\mathrm{Zn}$ and $\mathrm{Cu} / \mathrm{Zn}$ Superoxide Dismutase. Plos One 2011; 6 (2): e16938.

31. Shen SQ, Zhang Y, Xiang JJ, Xiong CL. Protective effect of curcumin against liver warm ischemia/reperfusion injury in rat model is associated with regulation of heat shock protein and antioxidant enzymes. World $\mathbf{J}$ Gastroenterol 2007; 13: 1953-1961.

32. Cetin A, Kaynar L, Kocyigit I et al. Role of grape seed extract on methotrexate induced oxidative stress in rat liver. Am J Chin Med 2008; 36: $861-872$

33. Xian C J, Howarth GS, Mardell CE et al. Temporal changes in TFF3 expression and jejunal morphology during methotrexate-induced damage and and repair. Am J Physiol 1999; 277: 785-795.

34. Jeynes BJ, Altmann GG. Light and scanning electron microscopic observations of the effects of sublethal doses of methotrexate on the rat small intestine. Anat Rec 1978; 191: 1-17.

35. Gao F, Tomitori H, Igarashi K, Horie T. Correlation between methotrexate induced intestinal damage and decrease in polyamine content. Life Sci 2002; 72: 669-676.

36. Sukhotnik I, Mogilner JG, Shteinberg D et al. Leptin accelerates enterocyte turnover during methotrexate-induced intestinal mucositis in a rat. Cancer Biol Ther 2009; 8: 899-906.

37. Fremont L. Biological effects of resveratrol. Life Sci 2000; 66: 663-673.

38. Tunali-Akbay T, Sehirli O, Ercan F, Sener G. Resveratrol protects against methotrexate-induced hepatic injury in rats. J Pharm Pharm Sci 2010; 13: 303-310.

39. Li ZD, Ma QY, Wang CA. Effect of resveratrol on pancreatic oxygen free radicals in rats with severe acute pancreatitis. World J Gastroenterol 2006; 12: 137-140.

40. Yildiz F, Terzi A, Coban S, et al.Protective effects of resveratrol on small intestines against intestinal ischemia-reperfusion injury in rats. $\mathrm{J}$ Gastroenterol and Hepatol 2009; 24: 1781-1785.

41. Karabulut AB, Kirimlioglu V, Kirimlioglu H, Yilmaz S, Isik B, Isikgil O. Protective effects of resveratrol on spleen and ileum in rats subjected to ischemia-reperfusion. Transplant Proc 2006; 38: 375-377.

Received April 3, 2013. Accepted March 20, 2015. 